\title{
Novel Mercaptan Derivatives Nanogold Particles with Hyperchromic and Fluorescence Sensitization Effects
}

\author{
Dorothy Araba Yakoba AGYAPONG ${ }^{1,3}$, Ran-Lin ZHAO ${ }^{1,2}$, \\ Cai-Xia LI ${ }^{1,2}$, Wen-Ju DU ${ }^{1,2}$ and Hong-Juan ZENG ${ }^{1,2,{ }^{*}}$ \\ ${ }^{1}$ Department of Biophysics, School of Life Science and Technology, University of \\ Electronic Science and Technology of China, Chengdu 610054, PR China \\ ${ }^{2}$ Center for Information in Biomedicine, University of Electronic Science and \\ Technology of China, Chengdu 610054, PR China \\ ${ }^{3}$ Biomedical Engineering Program, Kwame Nkrumah University of Science and \\ Technology, Kumasi, Ghana \\ ${ }^{*}$ Corresponding author
}

Keywords: Mercaptan derivatives nanogold particles, Carcinoembryonic antigen, Hyperchromic effect, Fluorescence sensitization effect.

\begin{abstract}
Carcinoembryonic antigen (CEA), typically associated with certain tumors is widely used as clinical tumor markers for diagnosing certain cancers and hence its detection is of great essence in clinical diagnostics. A large number of surface atoms of nanoparticles have huge surplus bonding ability, because of the surface effect of gold nanoparticles, resulting in reuniting and sinking among the nanoparticles which makes them unstable. However, for effective and efficient bioassay, a stable nanomaterial with good hyperchromic and fluorescence sensitization effects is of essence. In order to detect traces of CEA, a new kind of nanomaterial with hyperchromic and fluorescence sensitization effects is considered essential. In this paper, a new kind of mercaptan derivatives nanogold (MDN) particles is studied using ultraviolet-visible absorption spectra (UV-vis), fluorescence emission spectrum method. The prepared nanogold and MDN, was interacted with carcinoembryonic antigen (CEA). It was observed that the interaction between MDN and CEA had good hyperchromic and fluorescence sensitization effects. Given its promising advantage, it is expected that this nanomaterial with hyperchromic and fluorescence sensitization effects will be an attractive tool for use in many different fields.
\end{abstract}

\section{Introduction}

Carcinoembryonic antigen (CEA), is a kind of glycoprotein with molecular mass of about $200 \mathrm{kDa}$ and is a reliable and widely used tumor maker. It is involved in cell adhesion and normally produced during fetal development but the production stops before birth [1]. CEA, is also typically associated with certain tumors and is widely used as a clinical tumor marker for some familiar cancers [2]. CEA as a tumor-associated antigen has been identified as a biomarker for clinical diagnosis of colon tumors, breast tumors, ovarian carcinoma and cystadenocarcinoma [3]. Thus, 
monitoring the level of CEA in human serum is necessary in clinical assay since early cancer diagnosis is an important step for effective treatment and controlling of the disease. Therefore, the determination of CEA, plays an important role in diagnosis and clinical research.

In order to obtain clinically relevant results, it is essential to improve the sensitivity and enhance the signal. A series of strategies were employed to achieve this goal such as the use of secondary antibodies [4], functionalized nanoparticles [5], quantum dots [6] and atom transfer radical polymerization [7]. Due to their huge mass, high dielectric constant, and electromagnetic coupling between gold nanoparticles (AuNPs) and Au film, AuNPs have been used widely in a variety of works [8]. However a large number of surface atoms of nanoparticles, have huge surplus bonding abilities, because of the surface effect of gold nanoparticles, resulting in reuniting and sinking among the nanoparticles to make them unstable.

A kind of AuNP with hyperchromic and fluorescence sensitization effects is considered vital for the detection of traces of CEA. In this paper, novel mercaptan derivative of nanogold particles (MDN) are prepared and then studied using transmission electron microscopy (TEM), ultraviolet-visible absorption spectra (UV-vis), fluorescence emission (FE) spectrum and Fourier transform infrared spectrum (FT-IR) methods.

\section{Materials and Methods}

\section{Reagents and Materials}

Anti-CEA antibody and CEA standards were purchased from Biocell Biotech. Co. Ltd. (Zhengzhou, China). 1, 2-ethanedithiol and sodium borohydride $\left(\mathrm{NaBH}_{4}\right)$ were obtained from the First Reagent Factory (Shanghai, China). Tetrahydrofuran (THF) was purchased from $\mathrm{J} \& \mathrm{~K}$ Chemicals (Beijing, China). $\mathrm{HAuCl}_{4} .4 \mathrm{H}_{2} \mathrm{O}$ were purchased from Sinopharm Chemical Reagent Co. Ltd., (Shanghai, China). Phosphate buffer solution (PBS, $\mathrm{pH}$ 7.4) and other chemicals used were of analytical grade. All commercially obtained chemicals were used as received without further purification. All aqueous solutions were made using double distilled water, which was further purified to $18.2 \mathrm{M} \Omega$ with a $0.22 \mu \mathrm{m}$ millipore syringe filter.

\section{Preparation of MDN and MDN-anti-CEA Antibody Nanoparticles}

The MDN particles were prepared as described previously [9]. Briefly, $\mathrm{HAuCl}_{4}$ $(0.044 \mathrm{~g})$ was dissolved in anhydrous THF $(100 \mathrm{ml})$, followed by $0.5 \mathrm{ml}$ ethanedithiol addition. $\mathrm{NaBH}_{4}(5 \mathrm{ml})$ of freshly prepared $0.3 \mathrm{M}$ aqueous $\mathrm{NaBH}_{4}$ was added to the mixture dropwise with stirring. The reaction mixture turned blue, indicating the formation of gold nanoparticles. The stirring was continued for about $12 \mathrm{~h}$, and the functionalized gold nanoparticles were retrieved from THF using ultracentrifugation. The excess thiol was removed by successive washing with methanol. Prior to the experiment, thiol-derivative nanogold particles were diluted to 0.2 A 520 units $/ \mathrm{ml}$ with ethanol/deionized water, and the $\mathrm{pH}$ value was adjusted to between 9.0 and 9.5 using $\mathrm{Na}_{2} \mathrm{CO}_{3}$. After that, $200 \mu \mathrm{l}$ of anti-CEA (original 
concentration) was added to $5 \mathrm{ml}$ of the diluted gold colloids, and the mixture was incubated for $12 \mathrm{~h}$ at $4{ }^{\circ} \mathrm{C}$ with slight stirring $(150 \mathrm{rpm})$. The mixture was then centrifuged for $30 \mathrm{~min}$ at $4{ }^{\circ} \mathrm{C}(13,000 \mathrm{rpm})$. The obtained MDN-anti-CEA antibody was re-suspended in $5 \mathrm{ml}$ of $0.01 \mathrm{mg} \mathrm{ml}^{-1} \mathrm{BSA}$, and stored at $4{ }^{\circ} \mathrm{C}$ until further use.

\section{The Characterization of MDN and MDN-anti-CEA Antibody Nanoparticles}

UV-vis spectra of the samples were measured on a U-2910 spectrophotometer (Japan). Florescence spectra of the samples were measured on an F-4500 (Japan) spectrophotometer whiles the size of particles was investigated with a JEM-100CXII transmission electron microscope (TEM, Japan).

\section{Results}

\section{Transmission Electron Microscope (TEM) Characterization of Nanogold Particles and Mercaptan Derivatives Gold Nanoparticles}

The nanogold and MDNs were characterized using TEM technique. The results are as shown in Fig. 1. As can clearly be observed, the MDN's morphology was more regular with better dispersion than the nanogold particles.

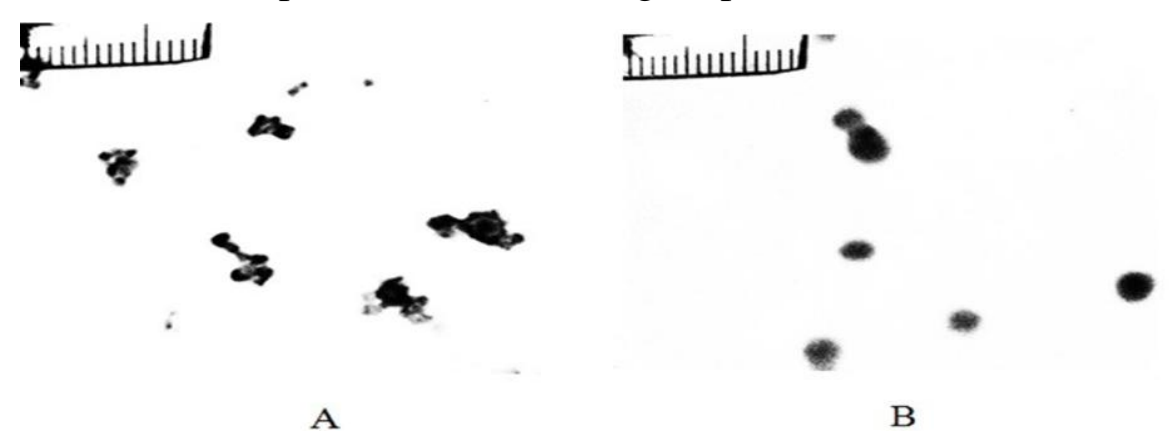

Figure 1. TEM images of nanogold particles (A) and mercaptan derivatives nanogold particles (B)

\section{The Ultraviolet Absorption Spectra of Nanogold Particles and Mercaptan Derivatives Nanogold Particles}

In the processing of mercaptan derivatives, the solution color changed from wine red to blue with the appearance of precipitates. UV-visible absorption spectrum test result (as shown in Fig. 2) clearly shows the maximum absorption peak position of nanometer gold appeared at $520 \mathrm{~nm}$, whiles that of the MDN appeared at $750 \mathrm{~nm}$ showing that the MDN particles were larger than the nanogold sol particle size.

\section{The Fluorescence Emission Spectra of Nanogold Particles and Mercaptan Derivatives Nanogold Particles}

Nanogold particles and MDN's fluorescence spectra are shown in Fig. 3. From the figure, under the activation of $520 \mathrm{~nm}$ wavelength of light that produced a strong fluorescence emission peak in a $787 \mathrm{~nm}$ and the strength increment was five times compared to AuNPs. 


\section{The Ultraviolet Absorption Spectra of the Mixed Solution of Nanogold and MDN Particles and CEA}

Fig. 4 shows the ultraviolet absorption spectra of tumor markers in CEA which existed in colorectal cancer, breast cancer and lung cancer and MDN-CEA solution for protein interaction testing.

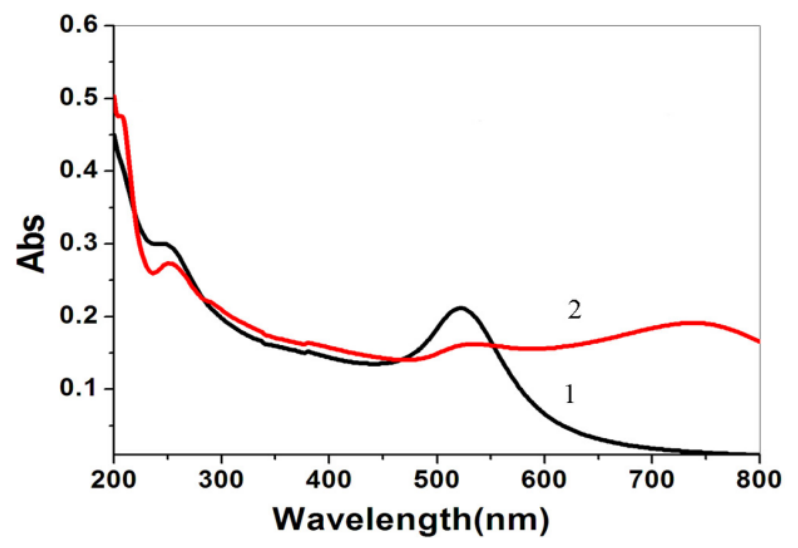

Figure 2. Ultraviolet absorption spectra of samples: nanogold particles (1), mercaptan derivatives nanogold particles (2).

\section{Discussion}

From the TEM images (Fig. 1), MDN's morphology was more regular with better dispersion than the nanogold particles, proving that MDN will have a better sensitivity than just the AuNPs [10]. UV-visible absorption spectrum test result (Fig. 2) clearly showed the maximum absorption peak position of AuNP appearing at $520 \mathrm{~nm}$, whiles that of the MDN appeared at $750 \mathrm{~nm}$ showing that the MDN particles were larger than the nanogold sol particle size. The MDN and nanogold solutions were mixed in mole ratio of 1:1 and the intensity of ultraviolet absorption was better than in the nanogold solution significantly which explains that mercaptan derivatives had hyperchromic effect on nanogold.

From Fig. 3, under the activation of $520 \mathrm{~nm}$ wavelength of light, a strong fluorescence emission peak in a $787 \mathrm{~nm}$ was produced for the MDN and the strength increment was five times compared to AuNPs. The fluorescence enhancement showed at the center of Au ion in MDN, exists more electron orbit by ligand transition to the center ion related orbit in the presence of new ligands oxalic mercaptan and hence increased fluorescence (fluorescence sensitization effect).

In order to test the viability of the MDN material in sensor applications, tumor markers in the carcinoembryonic antigen (CEA) which exists in colorectal cancer, breast cancer and lung cancer were used as the model, using the method of UV-vis spectroscopy of mercaptan derivatives nanogold and mercaptan derivatives nanogold and CEA protein interaction testing. From Fig. 4, mercaptan derivatives nanometer gold particles had no obvious effects on the protein structure and mixed with the CEA in solution, absorption peak did not change between 250-300 nm, which explains why mercaptan derivatives nanogold will not destroy the protein structure however between 200-250 nm exists a strong absorption peak indicating 
that mercaptan derivatives nanogold particles in CEA existed with good hyperchromic effect and increased the sensitivity of CEA detection. It can be observed that in a $530 \mathrm{~nm}$ exciting light, system in $783 \mathrm{~nm}$ markedly improved fluorescence emission peak after combining with the CEA protein, which explains an obvious fluorescence sensitization effect between MDN particles of target protein CEA.

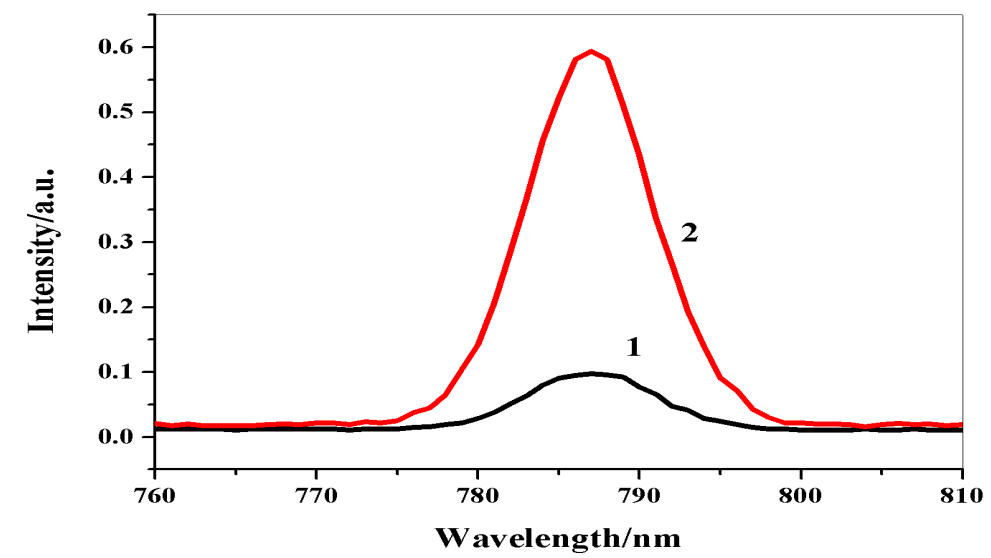

Figure 3. Fluorescence emission spectra of nanogold particles (1) and mercaptan derivatives nanogold particles (2)

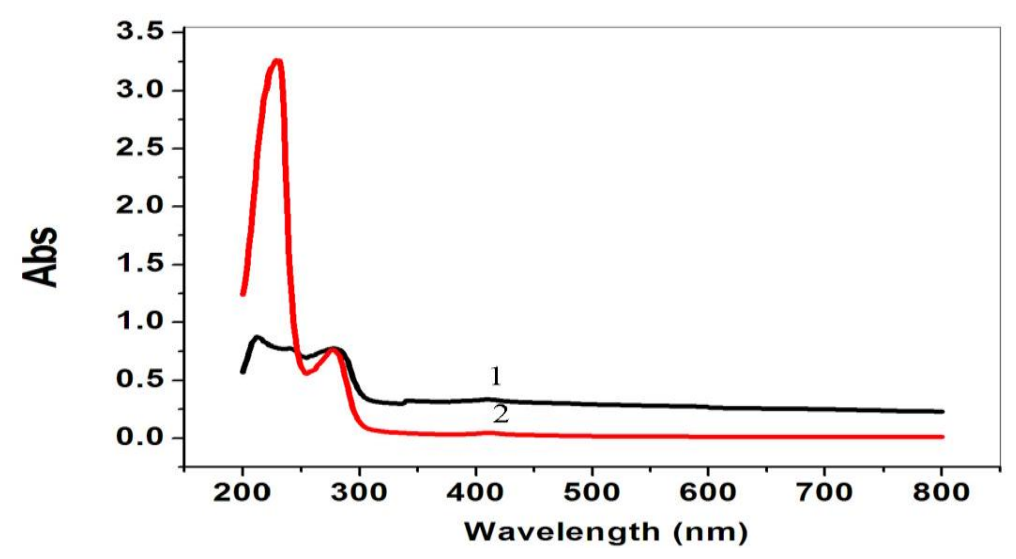

Figure 4. Ultraviolet absorption spectra of CEA solution (1) and solution of MDN particles and CEA (2)

\section{Conclusion}

The UV-vis and FE results show the presence of new ligands mercaptan, more electrons from the orbit of ligand which can excite to the central ion related orbits and increase the fluorescence of gold. Fluorescence sensitization effect was observed when MDN interacted with carcinoembryonic antigen (CEA) and no fluorescence sensitization effect was found when nanogold interacted with carcinoembryonic antigen (CEA). Due to its complexes in the presence of new gold thiol ligands, mercaptan derivatives nanogold particles reduced the forbidden band width of the Au ions, allowing more of the electron to move from the valence band into the conduction band with better absorption intensity. So, it can be concluded that the mercaptan derivatives nanogold particles are more suitable for protein 
detection since it had fluorescence sensitization. Therefore the novel mercaptan derivatives nanogold particles will have better biological detection application value than just nanogold.

\section{Acknowledgement}

The National Natural Science Foundation of China is appreciated for supporting this work financially via grant number 61071026.

\section{References}

[1] M. Grunnet, J.B. Sorensen, Carcinoembryonic antigen (CEA) as tumor marker in lung cancer, Lung Cancer 76 (2012)138-143.

[2] L. Hernández, A. Espasa, C. Fernández, A. Candela, C. Martín, S. Romero, CEA and CA 549 in serum and pleural fluid of patients with pleural effusion, Lung Cancer 36 (2002) 83-89.

[3] T. Iwazawa, T. Kanoh, S. Matsui, T. Monden, Diagnosis of lung cancer metastasis with CEA extracted from the dissected regional lymph nodes, Lung Cancer 29 (2000) 254.

[4] Y. Uludag, I. E. Tothill, Cancer Biomarker Detection in Serum Samples Using Surface Plasmon Resonance and Quartz Crystal Microbalance Sensors with Nanoparticle Signal Amplification, Anal. Chem. 84 (2012) 5898-5904.

[5] J. L. Wang, Z. Z. Zhu, A. Munir, H. S. Zhou, $\mathrm{Fe}_{3} \mathrm{O}_{4}$ nanoparticles-enhanced SPR sensing for ultrasensitive sandwich bio-assay, Talanta 84 (2011) 783-788.

[6] A. Shabani, M. Tabrizian, Design of a universal biointerface for sensitive, selective, and multiplex detection of biomarkers using surface plasmon resonance imaging, Analyst 138 (2013) 6052-6062.

[7] Y. Liu, Q. Cheng, Detection of Membrane-Binding Proteins by Surface Plasmon Resonance with an All-Aqueous Amplification Scheme, Anal. Chem. 84 (2012) 3179-3186.

[8] L. He, M. D. Musick, S. R. Nicewarner, F. G. Salinas, S. J. Benkovic, M. J. Natan, C. D. Keating, Colloidal Au-enhanced surface plasmon resonance for ultrasensitive detection of DNA hybridization, J. Am. Chem. Soc. 122 (2000) 9071-9077.

[9] M. P. Rowe, K. E. Plass, K. Kim, C. Kurdak, E.T. Zellers, A. J. Matzger, Single- phase synthesis of functionalized gold nanoparticles, Chem. Mater. 16 (2004) 3513-3517.

[10] D. J. Bray, S.G. Gilmour, F. J. Guild, A. C. Taylor, he effects of particle morphology on the analysis of discrete particle dispersion using Delaunay Tessellation, Composites Part A: Applied Science and Manufacturing 54 (2013) 37-45. 